\title{
Variação da capacidade funcional em idosos com estado depressivo e histórico de queda de residentes em uma instituição de longa permanência ${ }^{1}$
}

\author{
Jaqueline Colombo Ely", Adriana Arnt Brito*
}

\section{Resumo}

Esta pesquisa teve como objetivo geral identificar a variação da capacidade funcional em idosos com estado depressivo e histórico de queda residentes em uma instituição de longa permanência para idosos. Dentre os objetivos específicos incluiu-se identificar o grau de depressão em idosos por meio do Inventário de Beck para Depressão; verificar a capacidade funcional em idosos com estado depressivo e histórico de queda pelo Índice de Barthel e relacionar a idade, o sexo, o uso de medicamentos, o tempo de exposição à luz solar, a qualidade do sono e a realização de atividade física com a capacidade funcional dos idosos. Fizeram parte do estudo os idosos com idade igual ou superior a sessenta anos e que não se enquadravam nos critérios de exclusão da amostra, residentes em uma instituição de longa permanência de um município do interior do Rio Grande do Sul. Esta pesquisa foi do tipo quantitativa, explicativa e observacional. Os dados foram analisados utilizando o programa estatístico SPSS versão 14.0 para Windows. Ao analisar os dados, não se ve- rificou diferença estatística na variação da capacidade funcional em idosos com estado depressivo e histórico de queda. Constatou-se com as análises que não ocorreu variação estatística da capacidade funcional em idosos com essas características.

Palavras-chave: Idosos. Depressão. Queda. Sono. Atividade física.

\section{Introdução}

Segundo o Instituto Brasileiro de Geografia e Estatística (IBGE, 2004), a projeção da população no Brasil mostra a tendência de crescimento do número de idosos, que deverá alcançar os 34 milhões em 2025, o que colocará o Brasil na sexta posição mundial em número de idosos.

A preocupação com relação à capacidade funcional vem emergindo como destaque no campo da geriatria e gerontologia pelo fato de que a dependência

* Fisioterapeuta. Mestranda do Programa de Pós-Graduação em Envelhecimento Humano da Universidade de Passo Fundo. Endereço para correspondência: Jaqueline Colombo Ely, Rua Emílio Conrad, 585, CEP 95900-000, Lajeado - RS. E-mail: jcolomboely@yahoo.com.br

** Fisioterapeuta. Mestra em Desenvolvimento Regional pela Universidade de Santa Cruz do Sul. Professora do Centro de Ciências Biológicas e da Saúde do Centro Universitário Unidade Integrada Vale do Taquari de Ensino Superior.

1 O artigo científico é parte dos resultados encontrados no trabalho de conclusão do curso de Fisioterapia do Centro Universitário Univates.

$\hookrightarrow$ Recebido em agosto de 2008 - Avaliado em setembro de 2009.

$\rightarrow$ doi:10.5335/rbceh.2009.032 
funcional tende a se tornar um problema de saúde pública. (CALDAS, 2003). A capacidade funcional de idosos deve ser destacada como um processo dinâmico, reconhecendo-se que sua evolução pode ser modificada ou até mesmo prevenida, se houver assistência adequada.

A fisioterapia é um ramo da área da saúde que visa proporcionar uma melhor compreensão dos fatores associados à diminuição da capacidade funcional em idosos. A atuação profissional pode ser realizada no âmbito da atenção primária, secundária ou terciária à saúde. Cabe ao profissional fisioterapeuta preservar, manter, desenvolver ou restaurar a integridade físico-funcional dos indivíduos. (SULLIVAN; SCHMITZ, 2004). Em geriatria, a fisioterapia pode contribuir para um envelhecimento com saúde, qualidade de vida e maior independência funcional. (PEREIRA et al., 2002).

Os fatores associados à capacidade funcional têm sido amplamente estudados e relatados na literatura de fisioterapia. Dentre os fatores associados com a diminuição da capacidade funcional estão alguns problemas médicos (ROSA et al., 2003), como depressão (PENNINX et al., 2000) e histórico de queda. (GRAAFMANS et al., 1996; PERRACINI; RAMOS, 2002). Embora representem uma preocupação maior em saúde pública em virtude do seu impacto biopsicossocial (LEITE et al., 2006) e econômico (LIMA, 1999), os distúrbios depressivos nos idosos não foram extensivamente estudados. (VERAS, 1994). A queda não só afeta a capacidade funcional dos idosos como também demanda um grau excessivo de recursos financeiros no tratamento de suas consequências.
(GRISSO et al., 1992; RUBENSTEIN; POWERS; MACLEAN, 2001).

\section{Materiais e Métodos}

Trata-se de uma pesquisa do tipo observacional de abordagem quantitativa, realizada em uma instituição de longa permanência para idosos (ILPI) de um município do interior do Rio Grande do Sul.

Fizeram parte da pesquisa idosos residentes em uma ILPI, com idade igual ou superior a sessenta anos e as cuidadoras responsáveis pelos idosos. Excluíram-se do estudo os idosos permanentemente acamados, cadeirantes e com défice visual grave não corrigido; com distúrbios mentais que afetavam a capacidade perceptiva e de discernimento, analfabeto, analfabeto funcional e com défice auditivo grave não corrigido; idosos que não se comunicavam pela língua portuguesa e aqueles que não aceitaram participar do estudo. Dos 85 idosos residentes na ILPI com idade igual ou superior a sessenta anos, 53 foram excluídos da amostra por se enquadrarem nos critérios de exclusão. Restaram, dessa forma, 32 idosos passíveis de participar da pesquisa. Desses, sete idosos não quiseram participar do estudo, dois estavam ausentes no período em que se realizou a coleta de dados e um faleceu durante esse período. Assim, a amostra efetivamente estudada foi de 22 idosos.

Aplicaram-se neste estudo dois questionários e uma entrevista. Um dos questionários, direcionado a cada idoso, teve como propósito verificar seu estado depressivo, e o outro, direcionado à cuidadora responsável pelo idoso, teve como 
objetivo avaliar a capacidade funcional do mesmo. O Inventário de Beck para depressão foi o inquérito utilizado para o rastreamento de depressão e o Índice de Barthel foi o método utilizado na avaliação da capacidade funcional, pelo grau de independência nas atividades de vida diária. Na entrevista, o idoso respondeu a questões referentes ao histórico de queda, tempo de exposição à luz solar, qualidade do sono e realização de atividade física. As variáveis demográficas e uso de medicamentos foram obtidas por meio de informações contidas no prontuário médico dos idosos.

Os dados foram analisados utilizando-se o programa estatístico SPSS versão 14.0 para Windows. Realizou-se análise da correlação de Pearson entre as variáveis do estudo e análise de regressão sobre a capacidade funcional. Para todas as análises foi adotado o nível de significância de 0,05 .

O presente estudo iniciou-se após apreciação e aprovação pelo Comitê de Ética e Pesquisa da Univates (Coep/ Univates).

\section{Resultados}

O presente estudo avaliou 22 idosos, dos quais 11 (50\%) são do sexo feminino e $11(50 \%)$ do sexo masculino. As características demográficas dos idosos segundo histórico de queda e estado depressivo estão descritas na Tabela 1.

Tabela 1 - Características demográficas dos idosos segundo histórico de queda e estado depressivo.

\begin{tabular}{|c|c|c|c|c|c|c|}
\hline \multirow[b]{2}{*}{ Variáveis } & \multicolumn{2}{|c|}{ Histórico de queda } & \multicolumn{4}{|c|}{ Estado depressivo } \\
\hline & $\begin{array}{l}\text { Sem queda } \\
\quad(n=15)\end{array}$ & $\begin{array}{c}\text { Com queda } \\
(\mathrm{n}=7)\end{array}$ & $\begin{array}{l}\text { Mínimo } \\
(n=4)\end{array}$ & $\begin{array}{l}\text { Leve } \\
(\mathrm{n}=9)\end{array}$ & $\begin{array}{l}\text { Moderado } \\
\quad(n=7)\end{array}$ & $\begin{array}{l}\text { Grave } \\
(n=2)\end{array}$ \\
\hline $\begin{array}{l}\text { Média de idade } \\
\text { (desvio-padrão) }\end{array}$ & $\begin{array}{l}73,5 \\
(7,9)\end{array}$ & $\begin{array}{l}70,3 \\
(9,3)\end{array}$ & $\begin{array}{l}72,0 \\
(8,0)\end{array}$ & $\begin{array}{l}72,3 \\
(9,0)\end{array}$ & $\begin{array}{l}73,9 \\
(8,7)\end{array}$ & $\begin{array}{c}69,5 \\
(10,6)\end{array}$ \\
\hline $\begin{array}{c}\text { Masculino } \\
\mathrm{n}(\%)\end{array}$ & $\begin{array}{c}7 \\
(63,6)\end{array}$ & $\begin{array}{c}4 \\
(36,4)\end{array}$ & $\begin{array}{c}1 \\
(9,1)\end{array}$ & $\begin{array}{c}5 \\
(45,5)\end{array}$ & $\begin{array}{c}3 \\
(27,3)\end{array}$ & $\begin{array}{c}2 \\
(18,2)\end{array}$ \\
\hline $\begin{array}{c}\text { Feminino } \\
\mathrm{n}(\%)\end{array}$ & $\begin{array}{c}8 \\
(72,7)\end{array}$ & $\begin{array}{c}3 \\
(27,3)\end{array}$ & $\begin{array}{c}3 \\
(27,3)\end{array}$ & $\begin{array}{c}4 \\
(36,4)\end{array}$ & $\begin{array}{c}4 \\
(36,4)\end{array}$ & $\begin{array}{c}0 \\
(0,0)\end{array}$ \\
\hline
\end{tabular}

A média etária dos idosos residentes na ILPI foi de 72,5 e desvio-padrão de 8,3; a média etária dos idosos sem e com histórico de queda foi 73,5 , com desvio padrão de 7,9, e 70,3, com desvio-padrão de 9,3 , respectivamente. Não foi observada diferença estatisticamente significante no grupo com histórico de queda $(\mathrm{p}=$ 0,088). Do grupo de idosos sem histórico de queda, sete $(63,6 \%)$ são do sexo masculino e oito $(72,7 \%)$, do feminino. No grupo de idosos com histórico de queda, quatro $(36,4 \%)$ são do sexo masculino e três $(27,3 \%)$, do feminino.
A média etária dos idosos com estado depressivo mínimo foi de 72,0, com desvio-padrão de 8,0 anos; com depressão leve, 72,3, e desvio-padrão de 9,0 anos; com depressão moderada, 73,9, e desvio-padrão 8,7 anos. Nos idosos com depressão grave a média etária foi de 69,5 e desvio-padrão 10,6 anos. Não foi observada diferença estatisticamente significante no grupo com histórico de queda $(p=0,153)$. Do grupo de idosos com depressão mínima, um $(9,1 \%)$ é do sexo masculino e três $(27,3 \%)$, do feminino. No grupo de idosos com depressão 
leve, cinco $(45,5 \%)$ são do sexo masculino e quatro $(36,4 \%)$, do feminino. Nos idosos com depressão moderada, três $(27,3 \%)$ são do sexo masculino e quatro $(36,4 \%)$, do feminino. Naqueles com depressão grave, dois idosos $(18,18 \%)$ são do sexo masculino.
Ao se criarem diferentes faixas etárias para a variável idade, é possível observar algumas particularidades. A Tabela 2 mostra a distribuição na frequência da variável idade em faixas segundo histórico de queda e estado depressivo.

Tabela 2 - Distribuição na frequência da variável idade em faixas segundo histórico de queda e estado depressivo.

\begin{tabular}{ccccccc}
\hline \multirow{2}{*}{ Faixa etária } & \multicolumn{2}{c}{ Histórico de queda } & \multicolumn{4}{c}{ Estado depressivo } \\
\cline { 2 - 6 } & $\begin{array}{c}\text { Sem queda } \\
(\mathrm{n}=15)\end{array}$ & $\begin{array}{c}\text { Com queda } \\
(\mathrm{n}=7)\end{array}$ & $\begin{array}{c}\text { Mínimo } \\
(\mathrm{n}=4)\end{array}$ & $\begin{array}{c}\text { Leve } \\
(\mathrm{n}=9)\end{array}$ & $\begin{array}{c}\text { Moderado } \\
(\mathrm{n}=7)\end{array}$ & $\begin{array}{c}\text { Grave } \\
(\mathrm{n}=2)\end{array}$ \\
\hline De 60 a 69 anos & 4 & 4 & 1 & 4 & 2 & 1 \\
$\mathrm{n}(\%)$ & $(50,0)$ & $(50,0)$ & $(12,5)$ & $(50,0)$ & $(25,0)$ & $(12,5)$ \\
De 70 a 79 anos & 8 & 2 & 2 & 4 & 3 & 1 \\
$\mathrm{n}(\%)$ & $(80,0)$ & $(20,0)$ & $(20,0)$ & $(40,0)$ & $(30,0)$ & $(10,0)$ \\
De 80 a 89 anos & 3 & 1 & 1 & 1 & 2 & 0 \\
$\mathrm{n}(\%)$ & $(75,0)$ & $(25,0)$ & $(25,0)$ & $(25,0)$ & $(50,0)$ & $(0,0)$ \\
\hline
\end{tabular}

Nota: Análise de variância de uma via por meio do teste $\mathrm{F}$.

Quanto à faixa etária dos idosos sem histórico de queda, quatro (50\%) têm entre 60 e 69 anos; oito (80\%), entre 70 e 79 anos, e três (75\%), entre 80 e 89 anos. Nos idosos com histórico de queda, quatro $(50 \%)$ têm idade entre 60 e 69 anos; dois (20\%), entre 70 e 79 anos e um (25\%), entre 80 e 89 anos. Dos idosos com depressão mínima, um idoso $(12,5 \%)$ tem entre 60 e 69 anos; dois (20\%), entre 70 e 79 anos, e um (25\%), idade entre 80 e 89 anos. Nos idosos com depressão leve, quatro (50\%) têm idade entre 60 e 69 anos; quatro (40\%), entre 70 e 79 anos, e um (25\%), entre 80 e 89 anos. Nos idosos com depressão moderada, dois (25\%) têm idade entre 60 e 69 anos; três (30\%), entre 70 e 79 anos, e dois (50\%), entre 80 e 89 anos. Naqueles com depressão grave, um idoso $(12,5 \%)$ tem idade entre $60 \mathrm{e}$ 69 anos e um (10\%), entre 70 e 79 anos.

A Tabela 3 mostra a distribuição na frequência da variável estado depressivo e histórico de queda.

Tabela 3 - Distribuição na frequência da variável estado depressivo e histórico de queda.

\begin{tabular}{lcrcc}
\hline \multirow{2}{*}{ Nível de depressão } & \multicolumn{2}{c}{ Sem queda $(\mathrm{n}=15)$} & \multicolumn{2}{c}{ Com queda $(\mathrm{n}=7)$} \\
\cline { 2 - 5 } & $\mathrm{n}$ & $\%$ & $\mathrm{n}$ & $\%$ \\
\hline Mínima & 2 & 50,0 & 2 & 50,0 \\
Leve & 5 & 55,6 & 4 & 44,5 \\
Moderada & 6 & 85,7 & 1 & 14,3 \\
Grave & 2 & 100,0 & 0 & 0,0 \\
\hline
\end{tabular}

Nota: Análise de variância de uma via por meio do teste F.

Analisando a Tabela 3, verifica-se que, dos 15 idosos sem histórico de queda, dois (50\%) apresentaram depressão mínima, cinco $(55,6 \%)$, depressão leve; seis $(85,7 \%)$, depressão moderada e dois (100\%), depressão grave. Nos sete ido- 
sos com histórico de queda, dois (50\%) apresentaram depressão mínima; quatro $(44,5 \%)$, depressão leve e um $(14,3 \%)$, depressão moderada. A avaliação da capacidade funcional está apresentada na Tabela 4.

Tabela 4 - Distribuição na frequência das variáveis segundo faixas de capacidade funcional.

\begin{tabular}{|c|c|c|c|c|}
\hline \multirow[b]{2}{*}{ Variáveis } & \multirow[b]{2}{*}{ Categorias } & \multicolumn{3}{|c|}{ Faixas de capacidade funcional } \\
\hline & & Independência & $\begin{array}{c}\text { Dependência } \\
\text { leve }\end{array}$ & $\begin{array}{c}\text { Dependência } \\
\text { grave }\end{array}$ \\
\hline \multirow{3}{*}{ Histórico de queda } & Não & $9(60,0 \%)$ & $5(33,3 \%)$ & $1(6,7 \%)$ \\
\hline & Sim & $3(42,9 \%)$ & $4(57,1 \%)$ & - \\
\hline & Mínimo & $3(75,0 \%)$ & $1(35,0 \%)$ & - \\
\hline \multirow{4}{*}{ Estado depressivo } & Leve & $4(44,4 \%)$ & $5(55,6 \%)$ & - \\
\hline & Moderado & $5(71,4 \%)$ & $1(14,3 \%)$ & $1(14,3 \%)$ \\
\hline & Grave & - & $2(100,0 \%)$ & - \\
\hline & 60 a 69 anos & $5(62,5 \%)$ & $2(35,0 \%)$ & $1(12,5 \%)$ \\
\hline \multirow[t]{2}{*}{ Faixa etária } & 70 a 79 anos & $5(50,0 \%)$ & $5(50,0 \%)$ & - \\
\hline & 80 a 89 anos & $2(50,0 \%)$ & $2(50,0 \%)$ & - \\
\hline \multirow{2}{*}{ Sexo } & Masculino & $5(45,5 \%)$ & $5(45,5 \%)$ & $1(9,1 \%)$ \\
\hline & Feminino & $7(63,6 \%)$ & $4(36,4 \%)$ & - \\
\hline \multirow{2}{*}{ Medicamentos } & Não & $4(50,0 \%)$ & $4(50,0 \%)$ & - \\
\hline & Sim & $8(57,1 \%)$ & $5(35,7 \%)$ & $1(7,1 \%)$ \\
\hline \multirow{4}{*}{ Tempo de luz } & Menos de 2 horas & $1(20,0 \%)$ & $4(80,0 \%)$ & - \\
\hline & Entre 3 e 5 horas & $6(66,7 \%)$ & $2(22,292 \%)$ & $1(11,1 \%)$ \\
\hline & Entre 6 e 8 horas & $4(57,1 \%)$ & $3(42,9 \%)$ & - \\
\hline & Entre 9 e 11 horas & $1(100,0 \%)$ & - & - \\
\hline \multirow{2}{*}{ Sono } & Não & $7(63,6 \%)$ & $4(36,4 \%)$ & - \\
\hline & Sim & $5(45,5 \%)$ & $5(45,5 \%)$ & $1(9,1 \%)$ \\
\hline \multirow{2}{*}{ Atividade física } & Sim & $8(88,9 \%)$ & $1(11,1 \%)$ & - \\
\hline & Não & $4(30,8 \%)$ & $8(61,5 \%)$ & $1(7,7 \%)$ \\
\hline
\end{tabular}

Com relação à distribuição da frequência das variáveis do estudo segundo capacidade funcional em faixas, verificase que o Índice de Barthel classificou $60 \%(\mathrm{n}=9)$ dos indivíduos sem histórico de queda para independência; 33,3\% $(\mathrm{n}=5)$ para dependência leve e $6,7 \%$ (um idoso) para dependência grave. Nos indivíduos com histórico de queda observou-se $42,9 \%(\mathrm{n}=3)$ dos idosos para independência e $57,4 \%(n=4)$ para dependência leve.
O Índice de Barthel também classificou $75 \%(n=3)$ dos indivíduos com depressão mínima, para independência e $35 \%(\mathrm{n}=1)$ para dependência leve. Nos indivíduos com depressão leve observouse $44,4 \%(\mathrm{n}=4)$ para independência e $55,6 \%(\mathrm{n}=5)$ para dependência leve. Nos idosos com depressão moderada, observou-se $71,4 \%(\mathrm{n}=5)$ para independência, $14,3 \%(\mathrm{n}=1)$ para dependência leve e $14,3 \%(n=1)$ para dependência grave. Nos idosos com depressão grave, 
observou-se $100 \%(\mathrm{n}=2)$ para dependência leve.

Quanto à faixa de idade de 60 a 69 anos, o Índice de Barthel classificou $62,5 \%(\mathrm{n}=5)$ para independência, $35 \%$ $(\mathrm{n}=2)$ para dependência leve e $12,5 \%$ ( $\mathrm{n}=1$ ) para dependência grave. Com relação à faixa de 70 a 79 anos, obteve-se $50 \%(\mathrm{n}=5)$ para independência e 50\% $(\mathrm{n}=5)$ para dependência leve. Quanto à faixa de idade de 80 a 89 anos, o índice classificou $50 \%(\mathrm{n}=2)$ para independência e $50 \%(n=2)$ para dependência leve.

Com relação ao sexo masculino, $45,5 \%$ $(\mathrm{n}=5)$ dos idosos são independentes; $45,5 \%(n=5)$, dependentes leves e $9,1 \%$ $(\mathrm{n}=1)$, dependente grave. Quanto ao sexo feminino, $63,6 \%(\mathrm{n}=7)$ são independentes e $36,4 \%(\mathrm{n}=4)$, dependentes leves.

O Índice de Barthel também classificou $50 \%$ dos idosos $(n=4)$ que não fazem uso de medicamento para independência e $50 \%(n=4)$ para dependência leve. Daqueles que fazem uso de medicamento para dormir, $57,1 \%(\mathrm{n}=8)$ são independentes; 35,7\% ( $\mathrm{n}=5$ ), dependentes leves e $7,1 \%(\mathrm{n}=1)$, dependente grave. Com relação ao tempo de exposição à luz solar, o Índice de Barthel classificou 20\% $(\mathrm{n}=1)$ dos que se expõem por menos de duas horas diárias para independência e $80 \%(\mathrm{n}=4)$ para dependência leve. Daqueles que se expõem entre 3 e 5 horas diárias, $66,7 \%(\mathrm{n}=6)$ são independentes; $22,2 \%(\mathrm{n}=2)$, dependentes leves e $11,1 \%(\mathrm{n}=1)$, dependentes graves. Dos que se expõem entre 6 e 8 horas diárias, $57,1 \%(\mathrm{n}=4)$ são independentes e $42,9 \%$ $(\mathrm{n}=3)$, dependentes leves. Somente um idoso (100\%) se expõe entre 9 e 11 horas diárias, classificado, segundo a pontuação obtida no Índice de Barthel como idoso independente.

Com relação à qualidade do sono, o índice classificou $63,6 \%(\mathrm{n}=7)$ dos idosos sem problemas para dormir como independentes e $36,36 \%(n=4)$ para dependência leve. Dos idosos com modificação nos padrões de sono, $45,5 \%$ $(\mathrm{n}=5)$ são independentes; 45,5\% ( $\mathrm{n}=5)$, dependentes leves e $9,1 \%(n=1)$, dependentes graves.

Analisando a prática de atividade física, observa-se que o Índice de Barthel classificou 88,9\% ( $\mathrm{n}=8$ ) dos idosos que praticam atividade física regular para independência e $11,1 \%(n=1)$ para dependência leve. Dos idosos que não praticam atividade física regular, $30,8 \%(\mathrm{n}=4)$ são independentes; $61,5 \%$ $(n=8)$, dependentes leves e $7,7 \%(n=1)$, dependentes graves.

Antes de realizar as análises de regressão para mensurar o nível de dependência entre capacidade funcional e as demais variáveis, apresenta-se a matriz de correlação entre essas variáveis. Esta matriz tem como objetivo determinar o grau de dependência entre as variáveis analisadas com base no nível de significância. A matriz de correlação de Pearson, tendo como base as variáveis do estudo, aparece na Tabela 5.

Entre a variável dependente capacidade funcional e a variável independente prática de atividade física, observa-se uma alta correlação $(r=-0,520)$. É importante destacar que a correlação negativa entre a variável dependente e a variável independente capacidade funcional ocorre em razão da relação inversamente proporcional: à medida que a capacidade 
Tabela 5 - Matriz de correlação de Pearson entre as variáveis do estudo.

\begin{tabular}{ccccccccccc}
\hline & Idade & Sexo & UM & D & Queda & CF & Luz & Sono & AF & FE \\
\hline Sexo & $-0,017$ & & & & & & & & & \\
UM & $-0,643^{\star *}$ & 0,189 & & & & & & & & \\
D & $-0,003$ & $-0,260$ & $-0,157$ & & & & & & & \\
Queda & 0,187 & 0,098 & $-0,111$ & 0,361 & & & & & & \\
CF & $-0,103$ & 0,024 & 0,009 & $-0,275$ & $-0,187$ & & & & & \\
Luz & $-0,094$ & $-0,546^{\star *}$ & 0,052 & $-0,080$ & 0,032 & 0,419 & & & & \\
Sono & 0,332 & $-0,273$ & $-0,189$ & $0,469^{\star}$ & 0,293 & $-0,339$ & 0,109 & & & \\
AF & $-0,040$ & $-0,092$ & $-0,052$ & $-0,120$ & $-0,171$ & $-0,520^{*}$ & $-0,373$ & $-0,092$ & & \\
FE & $0,951^{* *}$ & 0,000 & $-0,588^{\star *}$ & $-0,053$ & 0,235 & $-0,048$ & $-0,097$ & 0,254 & $-0,082$ & \\
CFF & 0,055 & 0,252 & $-0,048$ & $-0,230$ & 0,025 & $0,883^{\star *}$ & 0,241 & $-0,252$ & $-0,501^{*}$ & 0,072 \\
\hline
\end{tabular}

Notas: UM - Uso de medicamentos; D - Depressão; CF - Capacidade funcional; AF - Atividade física; FE - Faixas etárias; CFF - Capacidade funcional.

*** Correlação é significante ao nível 0.01 .

* Correlação é significante ao nível 0.05 .

funcional aumenta, a prática de atividade física diminui. Fragmentando a variável dependente capacidade funcional em faixas, percebe-se, novamente, uma correlação estatisticamente significante $(r=-0,501)$.

Foram encontradas correlações significativas estatisticamente também para idade e uso de medicamentos $(\mathrm{r}=-0,0643)$, gênero e tempo de exposição à luz solar $(\mathrm{r}=-0,546)$, uso de medicamentos e faixa de idade $(r=-0,588)$ e estado depressivo e qualidade do sono $(r=0,469)$.

Analisando a variável capacidade funcional com o estado depressivo e histórico de queda, não se obtiveram dados estatisticamente significantes ( $p=0,598$ e $p=0,539$, respectivamente). Todavia, ao analisar a associação da variável dependente capacidade funcional com a variável independente atividade física, obteve-se significância estatística $(\mathrm{p}=0,022)$. Ao analisar a faixa etária por todas as outras variáveis, verificouse significância estatística apenas para a variável uso de medicamentos $(\mathrm{p}=$ $0,014)$. Ao analisar a relação entre o histórico de queda e estado depressivo, observa-se uma tendência à associação entre as duas variáveis $\left(p=0,099 ; R^{2}=\right.$ 0,$130 ; R^{2}$ ajustado $\left.=0,087\right)$. Não foi possível associar significativamente outras variáveis ao histórico de queda.

Tabela 6 - Análise de regressão sobre a capacidade funcional.

\begin{tabular}{cccccc}
\hline Constante & \multicolumn{2}{c}{ Coeficientes não padronizados } & \multicolumn{2}{c}{ Coeficientes padronizados } \\
\hline Prática de atividade & $\mathrm{B}$ & Erro padrão & Beta & $\mathrm{t}$ & $\mathrm{p}$ \\
\cline { 2 - 6 } física & $-0,735$ & 0,284 & $-0,501$ & $-2,588$ & 0,018 \\
\hline
\end{tabular}

Pode-se observar na Tabela 6 que o coeficiente de determinação ajustado $\left(\mathrm{R}^{2}=0,213\right)$ indica que $21 \%$ da variação da capacidade funcional são explicados pela prática de atividade física. Percebe- se, dessa forma, a relação entre capacidade funcional e prática de atividade física, demonstrada pela significância estatística $(p=0,018)$. 


\section{Discussão}

Considerando que tanto o estado depressivo (PENNINX et al., 2000) quanto o histórico de quedas (FABRICIO; RODRIGUES; COSTA JUNIOR, 2004) geram maior probabilidade de desenvolver incapacidades funcionais e levando em conta que os dois fatores são passíveis de intervenção, a identificação da capacidade funcional em idosos com esse perfil constituiu importante fator que sustentou a realização desta pesquisa. Ainda que a capacidade funcional constitua objeto de estudo bastante explorado dentro do enfoque clínico geriátrico, não foram encontrados na literatura estudos que identificassem a variação da capacidade funcional em idosos com estado depressivo e histórico de queda associado. Este estudo traz, portanto, informações relevantes acerca do tema.

Mesmo levando em consideração que o número total de idosos efetivamente estudado $(\mathrm{n}=22)$ correspondeu a tão somente $25 \%$ do total de idosos residentes na ILPI, é possível observar que os dados encontrados neste estudo vão ao encontro do que está relatado na literatura científica. Neste estudo, a percentagem de idosos com histórico de queda foi de $32 \%$ do total de idosos. No Brasil, cerca de $30 \%$ dos idosos caem ao menos uma vez ao ano, e somente $52 \%$ dos idosos não relataram nenhum evento de queda durante um seguimento de dois anos. (PERRACINI; RAMOS, 2002).

Cacciatore (1998) cita que, entre os principais fatores relacionados à depressão, apresentam-se variáveis demográficas, tais como idade avançada e sexo feminino. Neste estudo, a análise de variância pelo teste $\mathrm{F}$ mostra que não ocorreu diferença estatisticamente significante entre as faixas etárias criadas com relação ao estado depressivo $(\mathrm{p}=0,726)$.

Segundo Diogo, Neri e Cachioni (2004), sintomas depressivos representam potenciais fatores associados a quedas, pois alteram o nível de atenção do idoso, provocam perda de energia, diminuem a autoconfiança e aumentam a indiferença ao meio ambiente. Em contrapartida, o histórico de queda também parece estar associado com sintomas depressivos. Legters (2002) relata que a queda pode levar à insegurança e ao medo de sofrer um novo episódio, restringindo a mobilidade geral e proporcionando um isolamento social, fatores já relatados na literatura científica como preditores de depressão em idosos. (PENNINX et al., 2000). Neste estudo, a análise de variância pelo teste $\mathrm{F}$ mostra que não ocorreu diferença estatisticamente significante entre a variável histórico de queda e o estado depressivo $(p=0,407)$.

A prática da atividade física é capaz de beneficiar pessoas de todas as faixas etárias, mas é especialmente importante para os idosos, os quais apresentam um declínio físico-funcional geral associado ao processo de senescência ou senilidade. (DELIBERATO, 2002).

Apesar de apresentarem significância estatística, cabe salientar que algumas das correlações observadas neste estudo não parecem demonstrar relevância clínica, como é o caso da correlação entre idade e uso de medicamentos 
e sexo e tempo de exposição à luz solar. Embora não específicos do processo de envelhecimento, a falta de adaptação às perturbações emocionais, transtornos orgânicos e afetivos e utilização de medicamentos para transtornos depressivos podem causar alterações no padrão de sono. (BENETO, 2000). É importante salientar que a depressão pode ser um fator de risco para o desenvolvimento de declínio cognitivo, particularmente entre indivíduos idosos institucionalizados. (PARMELLE et al., 1991).

Neste estudo não se verificou diferença estatística na variação da capacidade funcional em idosos com estado depressivo e histórico de queda, além de não se ter observado correlação entre essas variáveis. Entretanto, há algumas limitações metodológicas a serem consideradas. A amostra reduzida pode ter contribuído para a ausência de diferença estatística entre as variáveis estado depressivo e histórico de queda e entre estas e a variável capacidade funcional.

Outra limitação verificada foi a diferença de procedimentos na aplicação dos questionários sobre capacidade funcional e estado depressivo. Aos idosos aplicouse o questionário para avaliação do estado depressivo e, à cuidadora responsável, o que verificou a capacidade funcional dos mesmos. O questionário autoaplicável sobre estado depressivo, mesmo impresso com fontes e espaços maiores, evitando a interferência da aluna pesquisadora, pode não ter correspondido ao real estado depressivo, uma vez que a função cognitiva do sujeito, pela sua capacidade perceptiva e de discernimento, não foi verificada de forma objetiva.
Além desse, o fato de alguns idosos utilizarem tratamento medicamentoso para depressão e/ou ansiedade pode ter mascarado o real estado depressivo do sujeito. Também podemos nos questionar quanto a uma possível influência do fator ordem de aplicação do Inventário de Beck para depressão e Formulário Geral do Idoso. Neste estudo, o Inventário de Beck para depressão foi respondido anteriormente ao Formulário Geral do Idoso. Por tratar-se de um questionário extenso, não se descarta a hipótese de que as respostas obtidas no formulário geral tenham sido afetadas por questões como cansaço e/ou confusão mental advindas do Inventário de Beck para depressão, anteriormente aplicado.

É importante ressaltar, no entanto, que não ocorreu erro de instrumento de rastreamento, uma vez que a medida de avaliação do estado depressivo utilizada neste estudo constituiu-se de um método objetivo e padronizado de avaliação geriátrica (DIOGO; NERI; CACHIONI, 2004), utilizado há mais de dez anos no Brasil. (GORENSTEIN; ANDRADE; ZUARDI, 1998).

Com relação à avaliação funcional, há também a hipótese de que as respostas obtidas no Índice de Barthel não tenham sido fidedignas, como forma de proteger o nome da instituição. Também é importante salientar que o instrumento de avaliação utilizado foi coerente, uma vez que o índice corresponde a um dos mais utilizados na prática clínica e possui níveis de validade e confiabilidade aceitáveis no idioma original. (SAINSBURY et al., 2005). 
Functional capacity variation in older adults with depression and fall history living in a long term care institution to elderly

\begin{abstract}
The general aim of this research was identify the variation of functional capacity in older adults with depression and fall history living in a long term care institution to elderly. Among the specific objectives was included the identification of the level of depression in old adults through the Beck depression inventory; the verification of functional capacity of old adults with depression and historical falls through the Barthel index and the connection to the age, genus, the time of exposure to light, the sleep quality and the realization of physical activity with the functional capacity of the old adults. In this study participated old adults wider or equal than 60 years old and they were not in exclusion criterion's sample, living in a long-permanence institution situated in a district of Rio Grande do Sul. This research was classification like quantitative, qualitative and observation. The data were analyzed with the statistics program SPSS version 14.0 for Windows. In analyze of data we did not verify statistic difference in functional capacity' variation in old adults with depression and historical falls. Through the analyses we concluded that did not occur functional capacity's statistic variation in this kind of old adults.
\end{abstract}

Key words: Old adults. Depression. Fall. Sleep. Physical activity.

\section{Referências}

BENETO, A. Sleep disorders in elderly. Epidemiology. Rev. Neurol., v. 30, n. 6, p. 581-586, 2000.

CACCIATORE, F. Morbidity patterns in aged population in Southern Italy: a survey sampling. Arch. Gerontol. Geriatr., v. 26, p. 201-213, 1998.

CALDAS, C. P. Envelhecimento com dependência: responsabilidades e demandas da família. Cadernos de Saúde Pública, Rio de Janeiro, v. 19, n. 3, p. 773-781, 2003.

DELIBERATO, P. C. P. Fisioterapia preventiva: fundamentos e aplicações. São Paulo: Manole, 2002.

DIOGO, M. J. D.; NERI, A. L.; CACHIONI, M. Saúde e qualidade de vida na velhice. Campinas: Alínea, 2004.

FABRICIO, S. C. C.; RODRIGUES, R. A. P.; COSTA JUNIOR, M. L. Causas e consequências de quedas de idosos atendidos em hospital público. Revista de Saúde Pública, Rio de Janeiro, v. 38, n. 1, p, 93-99, 2004.

GORENSTEIN, C.; ANDRADE, L. H. S. G.; ZUARDI, A. W. Escalas de avaliação clínica em psiquiatria e psicofarmacologia. Revista de Psiquiatria Clínica, São Paulo, v. 26, n. 4, p. 65-69, 1998.

GRAAFMANS, W. C. et al. Falls in the elderly: a prospective study of risk factors and risk profiles. Am. J. Epidemiol., v. 143, v. 11, p. 1129-1136, 1996.

GRISSO, J. A. et al. The impact of falls in an inner-city elderly African-American population. JAGS, v. 40, n. 7, p. 673-678, 1992.

INSTITUTO BRASILEIRO DE GEOGRAFIA E ESTATÍSTICA. Projeção da população do Brasil por sexo e idade para o período de 1980-2050 - Revisão 2004. Metodologia e Resultados. Disponível em: <www.ibge.gov. br>. Acesso em: 25 fev. 2008.

LEGTERS, K. Fear of falling. Physical Therapy, v. 82, n. 3, p. 264-272, 2002. 
LEITE, V. M. M. et al. Depressão e envelhecimento: estudo nos participantes do Programa Universidade Aberta à Terceira Idade. Revista de Saúde Brasileira Materno Infantil, Pernambuco, v. 6, n. 1, p. 31-38, 2006.

LIMA, M. S. Epidemiologia e impacto social. Revista Brasileira de Psiquiatria, São Paulo, v, 21, n. 1, p. 1-5, 1999.

PARMELLE, P. A et al. Depression and cognitive change among institutionalized aged. Psychol. Aging, v. 6, n. 4, p. 504-511, 1991.

PENNINX, B. W. J. H. et al. Changes in depression and physical decline in older adults: a longitudinal perspective. J. Affect Disorders, v. 61, n. 1-2, p. 1-12, 2000.

PEREIRA, L. S. M. et al. Fisioterapia. In: FREITAS, E. V. et al. Tratado de geriatria e gerontologia. Rio de Janeiro: Guanabara Koogan, 2002, p. 846-855.

PERRACINI, M. R.; RAMOS, L. R. Fatores associados a quedas em um coorte de idosos residentes na comunidade. Revista de Saúde Pública, Rio de Janeiro, v. 36, n. 6, p. 709-716, 2002.

ROSA, T. E. C. et al. Fatores determinantes na capacidade funcional de idosos. Revista de Saúde Pública, Rio de Janeiro, v. 37, n. 1, p. 40-48, 2003.

RUBENSTEIN, L. Z.; POWERS, C.; MACLEAN, C. H. Quality indicators for the management and prevention of falls and mobility problems in vulnerable elders. Ann. Intern. Med., v. 135, n. 8, p. 686-693, 2001.

SAINSBURY, A. et al. Reliability of the Barthel index when used with older people. Age and Ageing, v. 34, n. 3, p. 228-232, 2005.

SULLIVAN, S. B. O.; SCHMITZ, T. J. Fisioterapia: avaliação e tratamento. 4. ed. São Paulo: Manole, 2004.

VERAS, R. P. País jovem com cabelos brancos: a saúde do idoso no Brasil. 3. ed. Rio de Janeiro: Relume Dumará, 1994. 